\title{
CHARACTERIZATIONS OF COMPLETELY HAUSDORFF-CLOSED SPACES
}

\author{
LARRY L. HERRINGTON
}

\begin{abstract}
This paper gives several characterizations of completely Hausdorff-closed spaces.
\end{abstract}

1. Introduction. Our primary interest is the investigation of completely Hausdorff-closed spaces. Current characterizations of completely Hausdorffclosed spaces are given in terms of completely Hausdorff open filterbases [ 1 , p. 103]. In this paper we give characterizations of completely Hausdorffclosed spaces in terms of nets and arbitrary filterbases. These characterizations are obtained mainly through the introduction of a type of convergence for filterbases and nets that we call $f$-convergence.

Throughout, $\operatorname{cl}(A)$ will denote the closure of a $\operatorname{set} A$ and $\operatorname{Int}(A)$ will denote the interior of $A$. Also, we will use the letter $R$ to denote the real line.

2. Preliminary definitions and theorems. Let $f: X \rightarrow R$ be a continuous real-valued function from a topological space $X$ into the reals and let $p \in X$. If $H$ is an open set in $R$ containing $f(p)$, then we say that $f$ and $H$ is an ordered pair for $p$ and denote this by $(f, H)_{p}$.

Definition 2.1. Let $X$ be a topological space and let $F=\left\{A_{\alpha}: \alpha \in \Delta\right\}$ be a filterbase in $X$. Then $F f$-converges to $p \in X\left(F \rightarrow_{f} p\right)$ if for each ordered pair $(f, H)_{p}$ there exists an $A_{\alpha} \in F$ such that $A_{\alpha} \subset f^{-1}(H)$. The filterbase $F$ $f$-accumulates to $p \in X\left(F \propto_{f} p\right)$ if for each ordered pair $(f, H)_{p}$ and for each $A_{\alpha} \in F, A_{\alpha} \cap f^{-1}(H) \neq \varnothing$.

We note that a filterbase $F=\left\{A_{\alpha}: \alpha \in \Delta\right\} f$-converges to $p \in X$ if and only if for each ordered pair $(f, H)_{p}$ there exists $A_{\alpha} \in F$ such that $f\left(A_{\alpha}\right) \subset H$. Also $F f$-accumulates to $p \in X$ if and only if for each ordered pair $(f, H)_{p}$ and for each $A_{\alpha} \in F, f\left(A_{\alpha}\right) \cap H \neq \varnothing$.

Convergence and accumulation of filterbases in the usual sense, of course, imply $f$-convergence and $f$-accumulation, respectively. However, the converses do not hold as the next example shows.

EXAMPLE 2.2. Let $X=[0,6]$ have as a subbase the usual open sets together with the set $S=\{r: 1<r<5$ and $r$ is rational $\}$. Let $F=\left\{A_{n}: n \in N\right\}$ be a filterbase in $X$ (where $A_{n}=\{x: 3<x<3+1 / n$ and $x$ is irrational $\}$ ) and let $p=3$. The filterbase $F$ does not converge or accumulate in the usual sense to $p$ but $F \rightarrow_{f} p$ and $F \propto_{f} q$.

There are a number of theorems concerning $f$-convergence and $f$ accumulation whose statements parallel those of convergence and accumula-

Received by the editors January 17, 1975.

AMS (MOS) subject classifications (1970). Primary 54D20.

Key words and phrases. Completely Hausdorff-closed spaces. 
tion in the usual sense. We give a sample of some of these theorems but omit their straightforward proofs.

THEOREM 2.3. In a topological space $X$ the following properties hold:

(a) If $F$ is a filterbase in $X$ such that $F$-converges to $p \in X$, then $F$ $f$-accumulates to $p$ and, in completely Hausdorff spaces, at no point other tinan $p$.

(b) Let $F_{1}$ and $F_{2}$ be two filterbases in $X$ where $F_{2}$ is stronger than $F_{1}$. Then $F_{1} f$-accumulates to $p \in X$ if $F_{2} f$-accumulates to $p$.

(c) A filterbase $F_{1} f$-accumulates to $p \in X$ if and only if there exists a filterbase $F_{2}$ stronger than $F_{1}$ such that $F_{2} f$-converges to $p$.

(d) A maximal filterbase $M$ in $X$ f-accumulates to $p \in X$ if and only if $M$ $f$-converges to $p$.

(e) $X$ is completely Hausdorff if and only if each $f$-convergent filterbase in $X$ $f$-converges to exactly one point.

(f) If $X$ is completely regular, then a filterbase $F$ converges (accumulates) to $p \in X$ if and only if $F f$-converges (resp., $f$-accumulates) to $p$.

Definition 2.4. Let $X$ be a topological space and let $\phi: D \rightarrow X$ be a net in $X$. Then $\phi f$-converges to $p \in X\left(\phi \rightarrow_{f} p\right)$ if for each ordered pair $(f, H)_{p}$ there exists a $d \in D$ such that $\phi\left(T_{d}\right) \subset f^{-1}(H)$ (where $T_{d}=\{c \in D: d<c\}$ ). The net $\phi f$-accumulates to $p \in X\left(\phi \propto_{f} p\right)$ if for each ordered pair $(f, H)_{p}$ and for every $d \in D, \phi\left(T_{d}\right) \cap f^{-1}(H) \neq \varnothing$.

Of course, if $\phi: D \rightarrow X$ is a net in $X$, the family $F(\phi)=\left\{\phi\left(T_{d}\right): d \in D\right\}$ is a filterbase in $X$ and it is routine to verify that:

(a) $F(\phi) \rightarrow_{f} p \in X$ if and only if $\phi \rightarrow_{f} p$.

(b) $F(\phi) \propto_{f} p \in X$ if and only if $\phi \propto_{f} p$.

Conversely, every filterbase $F$ in $X$ determines a net $\phi: D \rightarrow X$ such that:

(a) $F \rightarrow_{f} p \in X$ if and only if $\phi \rightarrow_{f} p$.

(b) $F \propto_{f} p \in X$ if and only if $\phi \propto_{f} p$.

The construction of such a net is the same as that of [2, p. 213].

We next state a few theorems concerning $f$-convergence for nets.

TheOREM 2.5. In a topological space $X$ the following properties hold.

(a) If $\phi$ is a net in $X$ such that $\phi f$-converges to $p \in X$, then $\phi f$-accumulates to $p$, and in completely Hausdorff spaces, at no point other than $p$.

(b) $A$ net $\phi f$-accumulates to $p \in X$ if and only if there exists a subnet of $\phi$ $f$-converging to $p$.

(c) A universal net $\phi f$-accumulates to $p \in X$ if and only if $\phi f$-converges to $p$.

3. Filterbase and net characterizations of completely Hausdorff-closed spaces. An open filterbase $F$ in $X$ is completely Hausdorff if and only if for each $p \notin A(F)$ (where $A(F)$ denotes the set of accumulation points of $F$ ), there exists an open set $U$ containing $p, V \in F$, and a continuous real-valued function $f: X \rightarrow R$ such that $f(U)=\{0\}$ and $f(V)=\{1\}$ [1, p. 103].

Let $A=\left\{U_{\alpha}: \alpha \in \Delta_{1}\right\}$ and $B=\left\{V_{\beta}: \beta \in \Delta_{2}\right\}$ be covers of a space $X$. Then $B$ is a continuous refinement of $A$ if and only if for each $V_{\beta} \in B$, there is a $U_{\alpha} \in A$ and a continuous real-valued function $f: X \rightarrow R$ such that $f\left(V_{\beta}\right)=\{0\}$ and $f\left(X-U_{\alpha}\right)=\{1\}$. An open cover is completely Hausdorff if and only if it has a continuous refinement [1, p. 103]. 
A completely Hausdorff space $X$ is completely Hausdorff-closed if and only if $X$ is a closed set in every completely Hausdorff space in which it can be embedded [1].

LEMma 3.1. Let $F=\left\{O_{\alpha}: \alpha \in \Delta\right\}$ be an open completely Hausdorff filterbase on $X$. Then $A(F)=A_{f}(F)$ (where $A_{f}(F)$ denotes the set of $f$-accumulation points of $F$ ).

Proof. Clearly we only need to show that $A_{f}(F) \subset A(F)$. Therefore, suppose that $p \notin A(F)$. Then there exists an open set $U$ containing $p$, $O_{\alpha} \in F$, and a continuous $f: X \rightarrow R$ such that $f(U)=\{0\}$ and $f\left(O_{\alpha}\right)=\{1\}$. Let $H=\left\{x \in R:-\frac{1}{2}<x<\frac{1}{2}\right\}$. Then $f\left(O_{\alpha}\right) \cap H=\varnothing$ shows that $p \notin$ $A_{f}(F)$. Therefore, we conclude that $A(F)=A_{f}(F)$.

Lemma 3.2. Let $F=\left\{A_{\alpha}: \alpha \in \Delta\right\}$ be a filterbase on $X$ and let $p \in X$. Then $F$ does not $f$-accumulate to $p$ if and only if there exists an ordered pair $(f, H)_{p}$ and an $A_{\alpha} \in F$ such that $\operatorname{cl}\left(f\left(A_{\alpha}\right)\right) \cap \operatorname{cl}(H)=\varnothing$.

Proof. The straightforward proof follows from Definition 2.1 and the regularity of the reals.

THEOREM 3.3. Let $X$ be a completely Hausdorff space. Then the following are equivalent.

(a) $X$ is completely Hausdorff-closed.

(b) Each filterbase $F$ in $X f$-accumulates to some point $p \in X$.

(c) Each maximal filterbase $M$ in $X$ f-converges to some point $p \in X$.

Proof. (a) implies (b). Suppose there exists a filterbase $F=\left\{A_{\alpha}: \alpha \in \Delta\right\}$ in $X$ that does not $f$-accumulate in $X$. Then for each $x \in X$ there exists an ordered pair $\left(f_{x}, H_{x}\right)_{x}$ and some $A_{\alpha(x)} \in F$ such that $\operatorname{cl}\left(f_{x}\left(A_{\alpha(x)}\right)\right) \cap \operatorname{cl}\left(H_{x}\right)$ $=\varnothing$ according to Lemma 3.2. Since $R$ is normal, there exists an open set $B_{x} \supset \operatorname{cl}\left(f_{x}\left(A_{\alpha(x)}\right)\right)$ such that $\operatorname{cl}\left(B_{x}\right) \cap \operatorname{cl}\left(H_{x}\right)=\varnothing$. Let $K_{x}=f^{-1}\left(\operatorname{cl}\left(B_{x}\right)\right)$ and choose an open set $U_{x}$ containing $x$ such that $f\left(U_{x}\right) \subset H_{x}$. Then $U_{x} \cap K_{x}$ $=\varnothing$ and $\operatorname{Int}\left(K_{x}\right) \supset A_{\alpha(x)}$. Now by Theorem 4.1 of [2, p. 146] there exists a continuous $g_{x}: R \rightarrow R$ with the property that $g_{x}\left(\operatorname{cl}\left(H_{x}\right)\right)=\{0\}$ and $g_{x}\left(\operatorname{cl}\left(B_{x}\right)\right)$ $=\{1\}$. Consequently for each $x \in X$, there exist open sets $U_{x}$ and $G_{x}=X-$ $K_{x}$ containing $x$ and a continuous real-valued function $h_{x}=g_{x} f_{x}$ with the property that $h_{x}\left(U_{x}\right)=\{0\}$ and $h_{x}\left(X-G_{x}\right)=\{1\}$. Therefore, $\left\{G_{x}: x \in X\right\}$ is a completely Hausdorff open cover of $X$. Since $X$ is completely Hausdorffclosed, there exists a finite subcollection $\left\{G_{x_{i}}: i=1,2, \ldots, n\right\}$ such that $\cup_{i=1}^{n} \operatorname{cl}\left(G_{x_{i}}\right)=X$ according to Theorem 4.9 of [1, p. 103]. We note that for each $i, X-\operatorname{cl}\left(G_{x_{i}}\right)=\operatorname{Int}\left(K_{x_{i}}\right)$. Therefore,

$$
\varnothing=\bigcap_{i=1}^{n}\left(X-\operatorname{cl}\left(G_{x_{i}}\right)\right)=\bigcap_{i=1}^{n} \operatorname{Int}\left(K_{x_{i}}\right) \supset \bigcap_{i=1}^{n} A_{\alpha\left(x_{i}\right)}
$$

which is a contradiction. We conclude that $F f$-accumulates in $X$.

(b) implies (a). Let $F=\left\{O_{\alpha}: \alpha \in \Delta\right\}$ be an open completely Hausdorff filterbase on $X$. By Lemma 3.1 and hypothesis (b) we have that $A_{f}(F)$ $=A(F) \neq \varnothing$. Therefore, $X$ is completely Hausdorff-closed according to Theorem 4.9 of $[1$, p. 103]. 
(b) implies (c). Let $M$ be a maximal filterbase on $X$. Then $M f$-accumulates to some point $p \in X$ and, hence, $f$-converges to $p$ by Theorem 2.3(d).

(c) implies (b). Let $F$ be a filterbase in $X$. Then there exists a maximal filterbase $M$ in $X$ which is stronger than $F$. Since $M f$-converges to some point $p \in X, F f$-accumulates to $p$ according to Theorem 2.3(b),

Our discussion in the previous section showed that filterbases and nets are "equivalent" in the sense of $f$-convergence and $f$-accumulation. Thus, we can now characterize completely Hausdorff-closed spaces in terms of nets.

THEOREM 3.4. In a completely Hausdorff space the following are equivalent:

(a) $X$ is completely Hausdorff-closed.

(b) Each net in $X$ has an $f$-accumulation point.

(c) Each universal net $f$-converges.

REMARK 3.5. For each topological space $(X, T)$ there is a corresponding topological space $\left(X, T_{*}\right)$ called the semiregular space associated with $(X, T)$ $\left[1\right.$, p. 96]. The topology $T_{*}$ is generated by the regular open sets in $(X, T)$. We note that when a space $Y$ is regular, a map $f:(X, T) \rightarrow Y$ is continuous if and only if $f:\left(X, T_{*}\right) \rightarrow Y$ is continuous. Consequently, it follows that a space $(X$, $T)$ is completely Hausdorff if and only if $\left(X, T_{*}\right)$ is completely Hausdorff. Also, it is easy to see that a filterbase $F$ on $X f$-accumulates to a point $p$ in ( $X$, $T$ ) if and only if $F f$-accumulates to $p$ in $\left(X, T_{*}\right)$. With this in consideration we give the following theorem.

THEOREM 3.6. A space $(X, T)$ is completely Hausdorff-closed if and only if $\left(X, T_{*}\right)$ is completely Hausdorff-closed.

Proof. The result follows from Theorem 3.3 and Remark 3.5.

4. First countable completely Hausdorff spaces. A space $(X, T)$ is called first countable and minimal completely Hausdorff if $T$ is first countable and completely Hausdorff, and if no first countable topology on $X$ which is strictly weaker than $T$ is completely Hausdorff. $(X, T)$ is first countable and completely Hausdorff-closed if $T$ is first countable and completely Hausdorff, and $(X, T)$ is a closed subspace of every first countable completely Hausdorff space in which it can be embedded [1].

THeOREM 4.1. A first countable completely Hausdorff space $X$ is first countable and completely Hausdorff-closed if each countable filterbase on $X f$ accumulates to some point $p \in X$.

Proof. Assume that $X$ is not completely Hausdorff-closed. Then there exists a first countable completely Hausdorff space $Y$ and a homeomorphism $h: X \rightarrow h(X) \subset Y$ such that $h(X)$ is not closed in $Y$. Thus there exists a point $p \in Y-h(X)$ such that $p \in \operatorname{cl}(h(X))$. Let $\left\{U_{i}: i=1,2,3, \cdots\right\}$ be a countable base at $p$. Then $F=\left\{U_{i} \cap h(X): i=1,2, \cdots\right\}$ forms a countable open filterbase on $h(X)$. Since $h(X)$ is homeomorphic to $X, F f$-accumulates to some point $z \in h(X)$. It is clear that $F$ converges to $p$. Therefore, by Theorem 2.3(a), $p$ is equal to $z$ which is a contradiction. We conclude that $X$ is completely Hausdorff-closed. 
THEOREM 4.2. A first countable completely Hausdorff space $X$ is first countable and completely Hausdorff-closed if each sequence in $X$-accumulates to some point $p \in X$.

PROOF. The proof is similar to that of Theorem 4.1.

LeMma 4.3. Suppose that $X$ is a completely Hausdorff space with the property that every sequence in $X$ with a unique $f$-accumulation point is convergent. Then every sequence in $X$ has an $f$-accumulation point.

Proof. Suppose there is a sequence, $\left(x_{n}\right)$, in $X$ which has no $f$-accumulation point. Fix $p \in X$ and define a sequence, $\left(z_{n}\right)$, by $z_{n}=p$ if $n$ is odd and $z_{n}=x_{n / 2}$ if $n$ is even. Clearly $p$ is the unique $f$-accumulation point of $\left(z_{n}\right)$ and $\left(z_{n}\right)$ does not converge to $p$.

THEOREM 4.4. Let $(X, T)$ be a first countable completely Hausdorff space. Then $X$ is first countable and minimal completely Hausdorff if every sequence in $X$ with a unique $f$-accumulation point is convergent.

Proof. Suppose that $h:(X, T) \rightarrow(Y, \sigma)$ is a bijective continuous mapping onto a first countable completely Hausdorff space $(Y, \sigma)$. We need to show that $h^{-1}$ is continuous. Let $\left(y_{n}\right)$ be a sequence in $Y$ converging to $y \in Y$. If $p \in X$ is an $f$-accumulation point of the sequence $\left(h^{-1}\left(y_{n}\right)\right)$, then the continuity of $h$ shows that $h(p)$ is an $f$-accumulation point of $\left(y_{n}\right)$ and, therefore, since $Y$ is completely Hausdorff, $h(p)=y$. Thus $h^{-1}(y)$ is the unique $f$-accumulation point of $\left(h^{-1}\left(y_{n}\right)\right)$ which implies, by hypothesis, that $\left(h^{-1}\left(y_{n}\right)\right)$ converges to $h^{-1}(y)$. This shows that $h$ is continuous. We conclude that $(X, T)$ is first countable and minimal completely Hausdorff.

\section{REFERENCES}

1. M. P. Berri, J. R. Porter and R. M. Stephenson, Jr., A survey of minimal topological spaces, General Topology and its Relations to Modern Analysis and Algebra, III (Proc. Conf., Kanpur, 1968), Academia, Prague, 1971, pp. 93-114. MR 43 \#3985.

2. J. Dugundji, Topology, Allyn and Bacon, Boston, Mass., 1966. MR 33 \# 1824.

Department of Mathematics, University of arkansas at Pine Bluff, Pine Bluff, ARKansas 71601 